\title{
Extracting the spin polarizabilities of the proton by measurement of Compton double-polarization observables
}

D. Paudyal, ${ }^{1}$ P. P. Martel $\odot,{ }^{2,3, *}$ G. M. Huber, ${ }^{1}$ D. Hornidge, ${ }^{2}$ S. Abt,${ }^{4}$ P. Achenbach,,${ }^{3}$ P. Adlarson, ${ }^{3}$ F. Afzal,${ }^{5}$ Z. Ahmed, ${ }^{1}$ C. S. Akondi, ${ }^{6}$ J. R. M. Annand ${ }^{7}$ H. J. Arends, ${ }^{3}$ M. Bashkanov, ${ }^{8}$ R. Beck,${ }^{5}$ M. Biroth,${ }^{3}$ N. S. Borisov, ${ }^{9}$ A. Braghieri, ${ }^{10}$ W. J. Briscoe, ${ }^{11}$ F. Cividini, ${ }^{3}$ S. Costanza,${ }^{10}$ C. Collicott,,${ }^{12,13}$ A. Denig, ${ }^{3}$ M. Dieterle, ${ }^{4}$ E. J. Downie, ${ }^{11}$ P. Drexler,${ }^{3}$ M. I. Ferretti-Bondy, ${ }^{3}$ S. Gardner, ${ }^{7}$ S. Garni, ${ }^{4}$ D. I. Glazier, ${ }^{7}$ D. Glowa, ${ }^{14}$ I. Gorodnov, ${ }^{9}$ W. Gradl, ${ }^{3}$ S. Günther, ${ }^{4}$ G. M. Gurevich, ${ }^{15}$ D. Hamilton, ${ }^{7}$ L. Heijkenskjöld, ${ }^{3}$ A. Käser, ${ }^{4}$ V. L. Kashevarov,,${ }^{3,9}$ S. Kay, ${ }^{1}$ I. Keshelashvili, ${ }^{4}$ R. Kondratiev, ${ }^{15}$ M. Korolija, ${ }^{16}$ B. Krusche, ${ }^{4}$ A. B. Lazarev, ${ }^{9}$ J. M. Linturi, ${ }^{3}$ V. Lisin,,${ }^{15}$ K. Livingston, ${ }^{7}$ S. Lutterer, ${ }^{4}$ I. J. D. MacGregor, ${ }^{7}$ R. Macrae, ${ }^{7}$ J. Mancell, ${ }^{7}$ D. M. Manley, ${ }^{6}$ V. Metag,,${ }^{17}$ W. Meyer, ${ }^{18}$ R. Miskimen,,${ }^{19}$ E. Mornacchi, ${ }^{3}$ C. Mullen, ${ }^{7}$ A. Mushkarenkov, ${ }^{19,15}$ A. B. Neganov, ${ }^{9}$ A. Neiser, ${ }^{3}$ M. Oberle,${ }^{4}$ M. Ostrick, ${ }^{3}$ P. B. Otte, ${ }^{3}$ P. Pedroni, ${ }^{10}$ A. Polonski, ${ }^{15}$ A. Powell, ${ }^{7}$ S. N. Prakhov, ${ }^{3,20}$ A. Rajabi, ${ }^{19}$ G. Reicherz,,${ }^{18}$ G. Ron, ${ }^{21}$ T. Rostomyan, ${ }^{4}$ A. Sarty,${ }^{13}$ C. Sfienti, ${ }^{3}$ M. H. Sikora, ${ }^{14}$ V. Sokhoyan,,${ }^{3,11}$ K. Spieker, ${ }^{5}$ O. Steffen, ${ }^{3}$ I. I. Strakovsky, ${ }^{11}$ Th. Strub,${ }^{4}$ I. Supek, ${ }^{16}$ A. Thiel, ${ }^{5}$ M. Thiel, ${ }^{3}$ A. Thomas, ${ }^{3}$ M. Unverzagt, ${ }^{3}$ Yu. A. Usov, ${ }^{9}$ S. Wagner, ${ }^{3}$ N. K. Walford, ${ }^{4}$ D. P. Watts, ${ }^{8}$ D. Werthmüller, ${ }^{8}$ J. Wettig, ${ }^{3}$ L. Witthauer, ${ }^{4}$ M. Wolfes, ${ }^{3}$ and L. Zana ${ }^{22}$

(A2 Collaboration)

\author{
${ }^{1}$ University of Regina, Regina, Saskatchewan S4S 0A2, Canada \\ ${ }^{2}$ Mount Allison University, Sackville, New Brunswick E4L 1E6, Canada \\ ${ }^{3}$ Institut für Kernphysik, University of Mainz, D-55099 Mainz, Germany \\ ${ }^{4}$ Institut für Physik, University of Basel, CH-4056, Basel, Switzerland \\ ${ }^{5}$ Helmholtz-Institut für Strahlen- und Kernphysik, University of Bonn, D-53115 Bonn, Germany \\ ${ }^{6}$ Kent State University, Kent, Ohio 44242-0001, USA \\ ${ }^{7}$ SUPA School of Physics and Astronomy, University of Glasgow, Glasgow G12 8QQ, United Kingdom \\ ${ }^{8}$ Department of Physics, University of York, Heslington, York Y010 5DD, United Kingdom \\ ${ }^{9}$ Joint Institute for Nuclear Research, 141980 Dubna, Russia \\ ${ }^{10}$ INFN Sezione di Pavia, I-27100 Pavia, Italy \\ ${ }^{11}$ The George Washington University, Washington, DC 20052-0001, USA \\ ${ }^{12}$ Dalhousie University, Halifax, Nova Scotia B3H 4R2, Canada \\ ${ }^{13}$ Saint Mary's University, Halifax, Nova Scotia B3H 3C3, Canada \\ ${ }^{14}$ SUPA School of Physics and Astronomy, University of Edinburgh, Edinburgh EH9 3FD, United Kingdom \\ ${ }^{15}$ Institute for Nuclear Research, 125047 Moscow, Russia \\ ${ }^{16}$ Rudjer Boskovic Institute, HR-10000 Zagreb, Croatia \\ ${ }^{17}$ II. Physikalisches Institut, University of Giessen, D-35392 Giessen, Germany \\ ${ }^{18}$ Institut für Experimentalphysik, Ruhr Universität, 44780 Bochum, Germany \\ ${ }^{19}$ University of Massachusetts Amherst, Amherst, Massachusetts 01003, USA \\ ${ }^{20}$ University of California Los Angeles, Los Angeles, California 90095-1547, USA \\ ${ }^{21}$ Racah Institute of Physics, Hebrew University of Jerusalem, Jerusalem 91904, Israel \\ ${ }^{22}$ Thomas Jefferson National Accelerator Facility, Newport News, Virginia 23606, USA
}

(Received 18 September 2019; revised 7 July 2020; accepted 11 August 2020; published 25 September 2020)

\begin{abstract}
The Compton double-polarization observable $\Sigma_{2 z}$ has been measured for the first time in the $\Delta(1232)$ resonance region using a circularly polarized photon beam incident on a longitudinally polarized proton target at the Mainz Microtron. This paper reports these results, together with the model-dependent extraction of four proton spin polarizabilities from fits to additional asymmetry data using either a dispersion relation calculation or a baryon chiral perturbation theory calculations with the weighted average of these two fits resulting in $\gamma_{E 1 E 1}=-2.87 \pm 0.52, \gamma_{M 1 M 1}=2.70 \pm 0.43, \gamma_{E 1 M 2}=-0.85 \pm 0.72$, and $\gamma_{M 1 E 2}=2.04 \pm 0.43$, in units of $10^{-4} \mathrm{fm}^{4}$.
\end{abstract}

DOI: 10.1103/PhysRevC.102.035205

\footnotetext{
*martel@uni-mainz.de
}

Published by the American Physical Society under the terms of the Creative Commons Attribution 4.0 International license. Further distribution of this work must maintain attribution to the author(s) and the published article's title, journal citation, and DOI. 


\section{INTRODUCTION}

The electromagnetic interaction of a photon with a nucleon can be studied through Compton scattering experiments. It is best described using an effective Hamiltonian expanded in terms of the incident photon energy. Structure observables of these composite systems are experimentally accessible by elastically scattering real photons from the nucleon in real Compton scattering (RCS). Over decades, RCS has been established as a benchmark for understanding the ground-state properties of the nucleon, such as the magnetic moment. However, the leading-order properties that are sensitive to the internal quark dynamics of the nucleon are still poorly understood experimentally. This paper uses RCS in the $\Delta(1232)$ resonance region as a probe to understand some internal structure observables of a nucleon, the nucleon polarizabilities. These are fundamental properties that describe how its internal structure deforms under an applied electromagnetic field $[1,2]$.

The electromagnetic field of the photon induces transitions of certain definite multipolarities while attempting to deform the nucleon. The effective Hamiltonian at second order in incident photon energy, $E_{\gamma}$, depends on the electric and magnetic scalar polarizabilities, $\alpha_{E 1}$ and $\beta_{M 1}$, and at third order depends on the spin polarizabilities (SPs).

The third-order effective Hamiltonian term in the spindependent interaction is

$$
\begin{aligned}
H_{\mathrm{eff}}^{(3)}= & -4 \pi\left[\frac{1}{2} \gamma_{E 1 E 1} \vec{\sigma}(\vec{E} \times \dot{\vec{E}})+\frac{1}{2} \gamma_{M 1 M 1} \vec{\sigma}(\vec{H} \times \dot{\vec{H}})\right. \\
& \left.-\gamma_{M 1 E 2} E_{i j} \sigma_{i} H_{j}+\gamma_{E 1 M 2} H_{i j} \sigma_{i} E_{j}\right]
\end{aligned}
$$

where $\dot{\vec{E}}, \dot{\vec{H}}, E_{i j}$, and $H_{i j}$ are the partial derivatives with respect to time and space defined as $\dot{\vec{E}}=\partial_{t} \vec{E}, \dot{\vec{H}}=\partial_{t} \vec{H}$, $E_{i j}=\frac{1}{2}\left(\partial_{i} E_{j}+\partial_{j} E_{i}\right)$, and $H_{i j}=\frac{1}{2}\left(\partial_{i} H_{j}+\partial_{j} H_{i}\right)$, and $\gamma_{E 1 E 1}$, $\gamma_{M 1 M 1}, \gamma_{M 1 E 2}$, and $\gamma_{E 1 M 2}$ are the four SPs. The physics behind these leading-order SPs involves the excitation of the spin$\frac{1}{2}$ target nucleon to some intermediate state $\left(\Delta\right.$ or $\left.N^{\star}\right)$ via an electric or magnetic ( $E 1$ or $M 1)$ dipole transition and a successive de-excitation back to a spin- $\frac{1}{2}$ nucleon final state via an electric or magnetic dipole ( $E 1$ or $M 1)$ or quadrupole (E2 or $M 2)$ transition. These internal structure constants are manifestations of the spin structure of the nucleon, which parametrize the "stiffness" of the nucleon's spin against the electromagnetically induced deformations relative to the spin axis.

Measurements of two linear combinations of these four SPs - the forward spin polarizability, $\gamma_{0}[3,4]$, and the backward spin polarizability, $\gamma_{\pi}$ [5]-have been reported for the proton by several experiments. An extraction of the individual proton SPs was recently published via measurement of the double-polarization Compton asymmetry $-\Sigma_{2 x}-$ using a transversely polarized proton target at the Mainz Microtron (MAMI) [6] in conjunction with the $\gamma_{0}$ and $\gamma_{\pi}$ results and measurement of the beam-polarization Compton asymmetry $\Sigma_{3}$ performed at the LEGS facility [7]. This paper describes an improvement to the extraction of these proton SPs from the measurement of the double-polarization asymmetry $\Sigma_{2 z}$ using a longitudinally polarized proton target at MAMI. $\Sigma_{2 z}$ is defined as

$$
\Sigma_{2 z}=\frac{1}{P_{\text {circ }}^{\gamma} P_{z}^{t}}\left[\frac{\left(N_{+z}^{R}+N_{-z}^{L}\right)-\left(N_{+z}^{L}+N_{-z}^{R}\right)}{\left(N_{+z}^{R}+N_{-z}^{L}\right)+\left(N_{+z}^{L}+N_{-z}^{R}\right)}\right],
$$

where $N_{ \pm z}^{R}$ and $N_{ \pm z}^{L}$ are the normalized yield for right-handed and left-handed helicity states of the beam with the target polarized in the $\pm z$ direction, and $P_{\text {circ }}^{\gamma}$ and $P_{z}^{t}$ are the degrees of the photon beam circular polarization and target polarization, respectively.

\section{EXPERIMENTAL SETUP}

The experiment was performed in the A2 hall at MAMI [8,9], a facility composed of a cascade of three race track microtrons (RTM) that can provide both unpolarized and longitudinally polarized electron beams with energies up to $1.6 \mathrm{GeV}$ [8]. The longitudinally polarized electron beam was produced by irradiating a strained GaAsP III-V semiconductor with circularly polarized laser light [10]. A $180^{\circ}$ polarization flip was provided by reversing the helicity of the laser light with a Pockels cell at a rate of approximately $1 \mathrm{~Hz}$. A standard Mott polarimeter [11], installed near the MAMI accelerator cascade, was used for polarization measurements. The average beam polarization was $86.8 \pm$ $0.1 \%$ [12]. For this measurement, a $450 \mathrm{MeV}$ polarized electron beam passed through an alloy radiator of cobalt and iron, producing circularly polarized Bremsstrahlung photons. The photon polarization, $P_{\gamma}$, was determined by the helicity transfer relationship

$$
P_{\gamma}=P_{e} \frac{4 E_{\gamma} E_{e}-E_{\gamma}^{2}}{4 E_{e}^{2}-4 E_{\gamma} E_{e}+3 E_{\gamma}^{2}},
$$

where $P_{e}$ is the electron beam polarization, $E_{e}$ is the electron beam energy, and $E_{\gamma}$ is the energy of the radiated photon. $E_{\gamma}$ was determined by detecting the Bremsstrahlung electrons in the tagged photon spectrometer [13], and only photons in the energy range $E_{\gamma}=265-305 \mathrm{MeV}$ were used for this analysis. The previously mentioned flip of the electron beam polarization direction results in a flip of the photon beam helicity, which, given the relatively fast rate of $1 \mathrm{~Hz}$, provides the $\Sigma_{2 z}$ asymmetry relatively free of systematic effects. The photon beam was passed through a $2.5-\mathrm{mm}$-diameter lead collimator, resulting in a beam spot size of $9 \mathrm{~mm}$ on the longitudinally polarized frozen spin target (FST) [14] located in the center of the Crystal Ball spectrometer (CB) [15].

The FST used dynamic nuclear polarization, and its polarization was measured with a nuclear magnetic resonance coil; both are described in detail in Ref. [14]. Polarization of up to $80 \%$ and relaxation times of nearly $1000 \mathrm{~h}$ were achieved $[13,16]$, and the direction of proton polarization was reversed approximately once per week. While flipping the photon helicity is enough to produce the $\Sigma_{2 z}$ asymmetry, additionally reversing the target polarization is useful to further remove systematic effects. Polarization measurements were completed at the start and end of each data taking period for different polarization orientations. Corrections to the target polarization due to ice buildup on the NMR coil [17] were determined with $\pi^{0}$ asymmetries as well as comparisons of 
unpolarized and polarized total inclusive and $\pi^{0}$ cross sections [18]. To reflect inconsistencies between these methods, a liberal systematic error of $10 \%$ for the target polarization was utilized.

Data were collected during two beamtimes in 2014 and 2015 using the nearly $4 \pi$ CB-TAPS detector system [19]: the $\mathrm{CB}$ as a central calorimeter, and TAPS as a forward calorimeter. The CB consists of 672 optically isolated $\mathrm{NaI}(\mathrm{Tl})$ crystals with a truncated triangular pyramid shape arranged in two hemispheres. It covers about $94 \%$ of $4 \pi$ steradians and an angular range of $21^{\circ} \leqslant \theta \leqslant 159^{\circ}$ [7]. TAPS consists of 366 hexagonal $\mathrm{BaF}_{2}$ crystals and two inner rings totaling $72 \mathrm{PbWO}_{4}$ crystals and covers an angular range of $2^{\circ} \leqslant \theta \leqslant$ $20^{\circ}$ [20]. Charged particles were identified using energy deposition in the particle identification detector and tracked by a pair of multi-wire proportional chambers or TAPS-veto detectors and their corresponding calorimetric detector. Although the CB-TAPS system covers the angular range of $2-159^{\circ}$, there are regions near the entrance and exit through the detectors that are less efficient. These regions are: (i) the forward hole in the TAPS detector, $2-6^{\circ}$, and (ii) the backward hole in the $\mathrm{CB}, 150-159^{\circ}$. Fiducial cuts were applied to remove all the data from these angular regions of reduced detection efficiency.

\section{DATA ANALYSIS}

The Compton scattering channel, $\gamma p \rightarrow \gamma p$, has a simple final state, but it is very important to correctly identify background from competing reactions because its cross section is only about $1 \%$ of the cross section for the dominant $\pi^{0}$ photoproduction process. In addition, under certain conditions, $\pi^{0}$ photoproduction can mimic the Compton scattering signature if one of the $\pi^{0}$ decay photons escapes the detector, or if the electromagnetic showers from the two photons overlap due to finite angular resolution. The Compton channel was identified by selecting events having a total deposited energy above $40 \mathrm{MeV}$, where a single neutral and a single charged track are reconstructed, with the former in coincidence with a hit in the tagger. In order to remove uncorrelated events between CBTAPS and the photon tagger, the timing differences between the neutral track and hits in the tagger were checked against a $20 \mathrm{~ns}$ wide prompt (coincidence) window and a $910 \mathrm{~ns}$ wide random window split in two with one on either side of the prompt peak. The random sample was normalized by the relative window widths and subtracted from the prompt timing signal.

To eliminate competing backgrounds from coherent and incoherent Compton scattering and $\pi^{0}$ photoproduction off of nonhydrogen nuclei in the FST from the windows and shells of the cryostat material (mainly ${ }^{3} \mathrm{He} /{ }^{4} \mathrm{He},{ }^{12} \mathrm{C}$, and ${ }^{16} \mathrm{O}$ ), separate data were taken by inserting a carbon foam target with density $0.55 \mathrm{~g} / \mathrm{cm}^{3}$ into the same cryostat and the normalized yield was subtracted. A base scaling factor was determined by the ratio of live-time corrected tagger scalers for the butanol and carbon data sets. Comparison of $\pi^{0}$ photoproduction simulations with the data showed that a correction to this ratio of approximately $10 \%$ was necessary to account for a higher contribution from the helium in the target

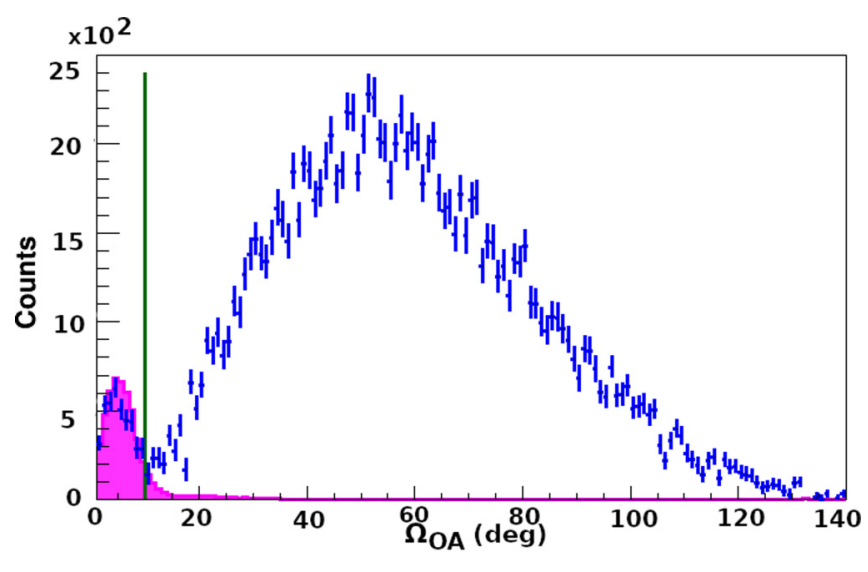

FIG. 1. Opening angle distribution for simulated Compton scattering events (magenta) compared with the carbon-subtracted data (blue) at $E_{\gamma}=285-305 \mathrm{MeV}$ and over all Compton angles. A cut of $10^{\circ}$ on the opening angle is indicated by the vertical line (green).

to this background. The carbon target density was chosen such that the number of nucleons equals the number of nonhydrogen nucleons from the ${ }^{3} \mathrm{He} /{ }^{4} \mathrm{He},{ }^{12} \mathrm{C}$, and ${ }^{16} \mathrm{O}$ in the target. To remove background from $\pi^{0}$ photoproduction off of the proton, the coincidence of a recoil charged track in addition to the neutral track was required, as mentioned above. However, since protons suffer a significant amount of energy loss when they travel from the event vertex through the target material, a ${ }^{3} \mathrm{He} /{ }^{4} \mathrm{He}$ refrigeration bath, various cryostat shells, and a longitudinal holding coil on their way to a detector crystal, the analysis was limited to an incident photon energy range of $E_{\gamma}=265-305 \mathrm{MeV}$. Further details on the background cuts, subtractions, and normalization factors can be found in Ref. $[12,18]$.

To identify events of interest, four-momentum conservation was used to constrain the observed reaction kinematics. As the background varies significantly across both energy and angle, their dependencies were studied. The tagged photon energy bins below $\gamma p \rightarrow \pi^{0} \pi^{0} p$ threshold were divided into five $\theta$ bins, and were analyzed separately. The opening angle $\left(\Omega_{\mathrm{OA}}\right)$, defined as the angle between the detected proton, $\vec{p}_{\text {recoil }}$, and where the proton was expected assuming RCS kinematics, $\vec{p}_{\text {miss }}=\vec{p}_{\gamma_{i}}-\vec{p}_{\gamma_{f}}, \cos \left(\Omega_{\mathrm{OA}}\right)=\frac{\vec{p}_{\text {miss }} \cdot \vec{p}_{\text {recoil }}}{\left|\vec{p}_{\text {miss }}\right| \times\left|\vec{p}_{\text {recoil }}\right|}$, was used for a two-body reaction selection. The Monte Carlo simulated opening angle results show a sharp peak around $5^{\circ}$, which is in good agreement with the data. The large background, as seen in Fig. 1, is mainly due to the $\pi^{0}$ photoproduction process from the proton. This can be suppressed by applying a $10^{\circ}$ opening angle cut, as indicated by the green vertical line. The Compton coplanarity angle, defined as the difference in the azimuthal angles of a scattered photon and a recoil proton, $\Delta \phi=\left|\phi_{\gamma f}-\phi_{p}\right|$, was used to suppress additional background. A cut on the fixed coplanarity angle, $\Delta \phi=180 \pm 15^{\circ}$, as indicated by the two vertical green lines in Fig. 2, was applied to the reconstructed events. For those events with a single neutral and a single charged track, the missing mass is calculated with

$$
M_{\text {miss }}^{2}=\left(E_{\gamma_{i}}+m_{p} c^{2}-E_{\gamma_{f}}\right)^{2}-\left(\vec{p}_{\gamma_{i}}-\vec{p}_{\gamma_{f}}\right)^{2} c^{2},
$$




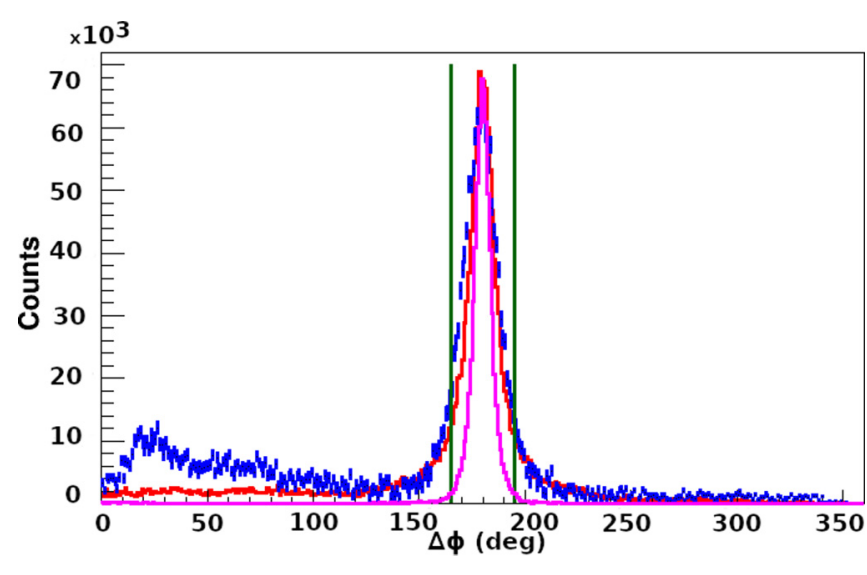

FIG. 2. Coplanarity distribution for simulated Compton scattering events (magenta), and simulated $\pi^{0}$ events that were analyzed as if they were a Compton photon (red), compared with the carbonsubtracted data (blue) at $E_{\gamma}=285-305 \mathrm{MeV}$ and over all Compton angles ( $\Omega_{\mathrm{OA}}$ cut from Fig. 1 is applied).

where $\left(E_{\gamma_{i}}, \vec{p}_{\gamma_{i}} c\right)$ and $\left(E_{\gamma_{f}}, \vec{p}_{\gamma_{f}} c\right)$ are the four vectors of the incident and scattered photon, respectively, and $m_{p}$ is the proton mass.

The carbon-subtracted $M_{\text {miss }}$ spectrum using the corrected carbon target scaling factors $[12,18]$ is shown in Fig. 3. Simulations of both Compton scattering and $\pi^{0}$ production were passed through the same analysis chain, with the same cuts applied. The distributions from these two reactions were added together (according to their known cross section at a given energy and angle). From these spectra, there is clearly good agreement of the data with the expected distribution up to $M_{\text {miss }} \approx 980 \mathrm{MeV} / c^{2}$. It is observed that $\pi^{0}$ photoproduction is the major source of background above a $M_{\text {miss }}$ of approximately $940 \mathrm{MeV} / c^{2}$, and hence it is necessary to set a clear upper $M_{\text {miss }}$ limit that coincides with the turn-on point of this background.

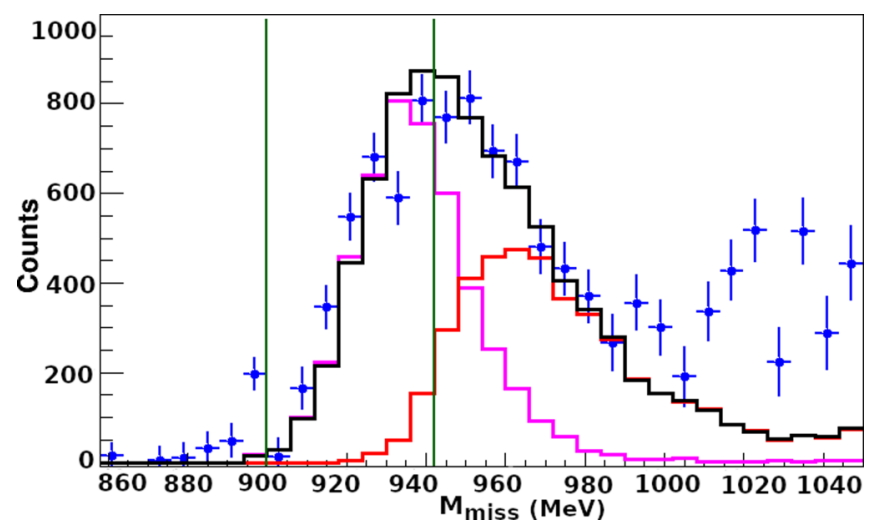

FIG. 3. Missing mass spectrum for carbon-subtracted data (blue), Monte Carlo simulated results from Compton scattering (magenta), and $\pi^{0}$ photoproduction (red) satisfying Compton cuts, and the sum of the two simulated contributions to show an expected distribution (black), all at $\theta_{\gamma}=125-140^{\circ}$ and $E_{\gamma}=285-305 \mathrm{MeV}$. Two vertical lines (green) represent the missing mass integration limit.
TABLE I. Summary of results and uncertainties for the Compton $\Sigma_{2 z}$ asymmetry.

\begin{tabular}{ccccc}
\hline \hline$E_{\gamma}(\mathrm{MeV})$ & $\theta_{\gamma}$ & $\Sigma_{2 z}$ & Rand. & Syst. \\
\hline \multirow{4}{*}{$265-285$} & $87.5^{\circ}$ & 0.193 & \pm 0.056 & \pm 0.024 \\
& $102.5^{\circ}$ & 0.290 & \pm 0.040 & \pm 0.035 \\
& $117.5^{\circ}$ & 0.402 & \pm 0.037 & \pm 0.048 \\
& $132.5^{\circ}$ & 0.672 & \pm 0.036 & \pm 0.077 \\
& $147.5^{\circ}$ & 0.672 & \pm 0.042 & \pm 0.081 \\
$285-305$ & $87.5^{\circ}$ & 0.121 & \pm 0.040 & \pm 0.016 \\
& $102.5^{\circ}$ & 0.279 & \pm 0.034 & \pm 0.033 \\
& $117.5^{\circ}$ & 0.428 & \pm 0.038 & \pm 0.048 \\
& $132.5^{\circ}$ & 0.591 & \pm 0.029 & \pm 0.066 \\
\hline \hline
\end{tabular}

Though $M_{\text {miss }}$ spectra can be integrated up to the most conservative limit of $938 \mathrm{MeV} / c^{2}$ (proton mass), the following steps were taken to maximize the integrated yield. First, the lower $M_{\text {miss }}$ limit was fixed at $900 \mathrm{MeV} / c^{2}$ for each energy and angle bin. Second, the asymmetry determined using the conservative $M_{\text {miss }}$ upper limit of $938 \mathrm{MeV} / c^{2}$ was taken as a reference. Finally, the asymmetry was allowed to vary a maximum of $5 \%$ by moving the $M_{\text {miss }}$ upper limit to higher values compared to the reference. This $\pm 5 \%$ is based on the systematic uncertainties from the choice of carbon target length and the ratio of $\pi^{0}$ photoproduction background to Compton scattering determined from simulation. As the asymmetry shifts either up or down for different bins, there is no concern about introducing a systematic shift from the 'correct' asymmetry. As an additional check the central value on the spin polarizabilities, as extracted by the method described below, were compared between the reference and final asymmetries, which indicated only small effects on $\gamma_{E 1 E 1}$ and $\gamma_{M 1 E 2}$ of approximately $20 \%$ of their errors and negligible effects on the other two. The resulting final $M_{\text {miss }}$ upper limits are between $940-948 \mathrm{MeV} / c^{2}$, and further details on this work can be found in Ref. [12,18].

\section{RESULTS}

The $\Sigma_{2 z}$ asymmetries for $E_{\gamma}=265-285 \mathrm{MeV}$ and $E_{\gamma}=$ 285-305 MeV, obtained by combining the results from the two beam times via their weighted average, are tabulated in Table I and shown in Fig. 4 along with determinations at $0^{\circ}$ through dispersive sum rules [21,22]. While the absolute statistical errors only vary between $0.029-0.056$, the relative errors vary between 5-33\% due to the small asymmetry at $90^{\circ}$. The systematic errors from the three different sources: target polarization $(10 \%)$, beam polarization $(2.7 \%)$, and carbon subtraction (3-6\%), were added in quadrature and their average between the 2014 and 2015 beam times for each Compton angle is listed in the table and shown as a separate block above the horizontal axis in the figures. These total systematic errors vary between $0.016-0.085$ absolute, or $11-13 \%$ relative. To study the sensitivity of the $\Sigma_{2 z}$ results on the SPs, a fixed- $t$ dispersion relation code (HDPV) [2,23,24] was used to generate predicted asymmetries at fixed laboratory energies for 

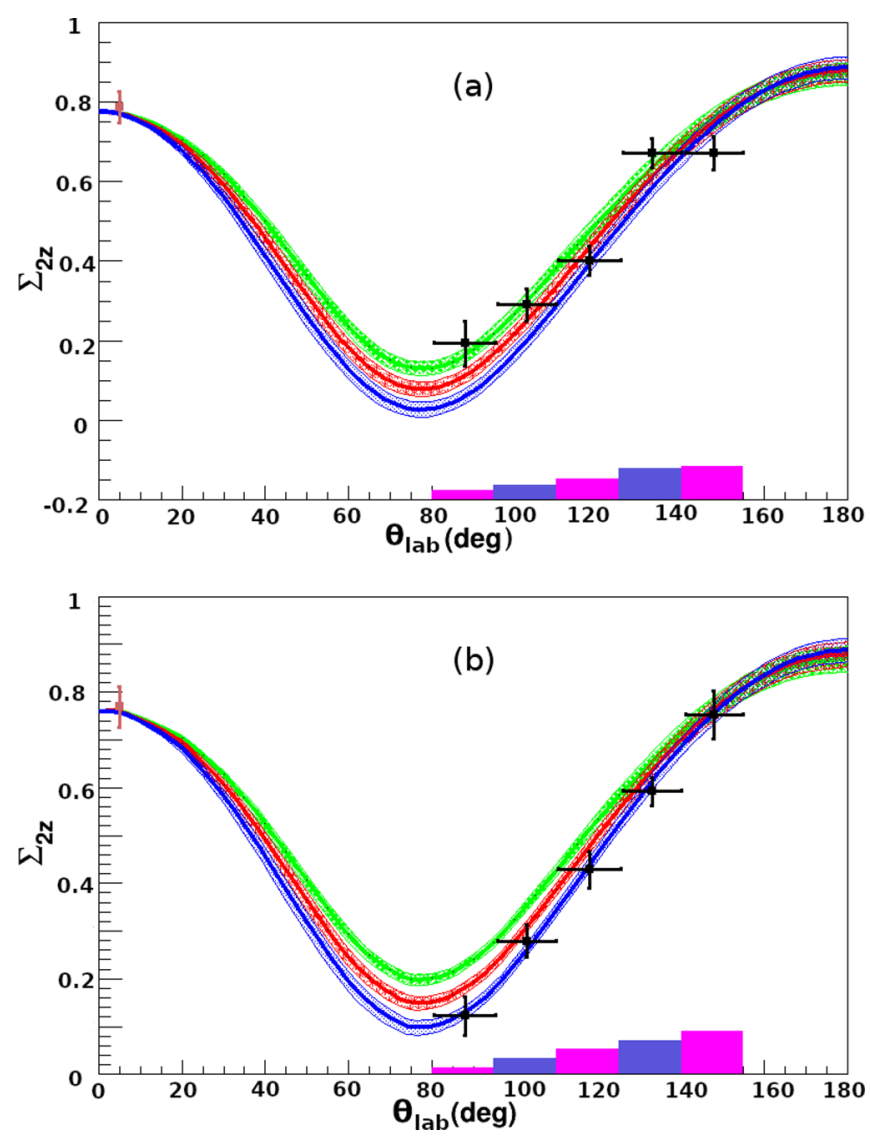

FIG. 4. Compton $\Sigma_{2 z}$ for $E_{\gamma}=265-285 \mathrm{MeV}$ (a) and $E_{\gamma}=$ 285-305 MeV (b). The red point is the value for $\Sigma_{2 z}$ at $0^{\circ}$, plotted at $5^{\circ}$ for readability, as determined by dispersive sum rules [21,22]. The curves are from the HDPV dispersion theory calculation of Pasquini et al., [2,23,24], where $\gamma_{E-}$ [27] is fixed at $-3.5 \times 10^{-4} \mathrm{fm}^{4}$ and $\gamma_{M-}$ [27] is set at $-0.5,1.5$, or $3.5 \times 10^{-4} \mathrm{fm}^{4}$, in the green, red, or blue bands, respectively. The width of each band represents the other parameters, $\gamma_{0}, \gamma_{\pi}, \alpha_{E 1}+\beta_{M 1}$, and $\alpha_{E 1}-\beta_{M 1}$ varying within their experimental errors. The error bars shown are point-to-point statistical plus random systematic errors added in quadrature. The correlated systematic uncertainties are shown as a separate block above the horizontal axis for each Compton angle.

various values of the scalar and spin polarizabilities. Predictions within baryon chiral perturbation theory $(\mathrm{B} \chi \mathrm{PT})$ [25] and heavy baryon chiral perturbation theory $(\mathrm{HB} \chi \mathrm{PT})[26,27]$ are also available, but are not shown in Fig. 4 to preserve readability. The code used nominal values for the scalar polarizabilities of $\alpha_{E 1}+\beta_{M 1}=13.8 \pm 0.4$ (Baldin sum rule) [28] and $\alpha_{E 1}-\beta_{M 1}=8.7 \pm 0.7$ (in units of $10^{-4} \mathrm{fm}^{3}$ ) [29], and for the SPs of $\gamma_{0}=-0.929 \pm 0.105[21,22]$ and $\gamma_{\pi}=8 \pm 1.8$ (in units of $10^{-4} \mathrm{fm}^{4}$ ) [5]. It should be noted that the value for $\alpha_{E 1}-\beta_{M 1}$ was chosen as the current PDG numbers [29], despite the debate regarding them [30,31], as the focus of this study is on the spin polarizabilities. It should also be noted that this value for $\gamma_{\pi}$ does not include the $\pi^{0}$-pole component, set as $-46.7 \times 10^{-4} \mathrm{fm}^{4}[31]$ in all of these studies.

Though $\gamma_{0}$ and $\gamma_{\pi}$ can form a basis of the SPs with $\gamma_{E 1 E 1}$ and $\gamma_{M 1 M 1}$, they can alternatively form an orthogonal basis with $\gamma_{E-}=\gamma_{E 1 E 1}-\gamma_{E 1 M 2}$ and $\gamma_{M-}=\gamma_{M 1 M 1}-\gamma_{M 1 E 2}$ [27].
TABLE II. Polarizabilities in $10^{-4} \mathrm{fm}^{4}$ from fitting $\Sigma_{2 z}, \Sigma_{2 x}$, and $\Sigma_{3}^{\text {LEGS }}$ asymmetries using either a HDPV [2,23,24] or a B $\chi$ PT [25] calculation, and weighted average of the SPs.

\begin{tabular}{lrrr}
\hline \hline & \multicolumn{3}{c}{$\Sigma_{2 z}, \Sigma_{2 x}$, and $\Sigma_{3}^{\text {LEGS }}$ data fits } \\
\cline { 2 - 4 } & \multicolumn{1}{c}{ HDPV } & \multicolumn{1}{c}{$\mathrm{B} \chi \mathrm{PT}$} & Weighted average \\
\hline$\gamma_{E 1 E 1}$ & $-3.18 \pm 0.52$ & $-2.65 \pm 0.43$ & $-2.87 \pm 0.52$ \\
$\gamma_{M 1 M 1}$ & $2.98 \pm 0.43$ & $2.43 \pm 0.42$ & $2.70 \pm 0.43$ \\
$\gamma_{E 1 M 2}$ & $-0.44 \pm 0.67$ & $-1.32 \pm 0.72$ & $-0.85 \pm 0.72$ \\
$\gamma_{M 1 E 2}$ & $1.58 \pm 0.43$ & $2.47 \pm 0.42$ & $2.04 \pm 0.43$ \\
$\chi^{2} /$ dof & 1.14 & 1.36 & \\
\hline \hline
\end{tabular}

In Fig. 4, $\gamma_{E-}$ was fixed at $-3.5 \times 10^{-4} \mathrm{fm}^{4}$ and $\gamma_{M-}$ was set at $-0.5,1.5$, or 3.5 in the same units. The various bands represent the different values for $\gamma_{M-}$, while the spread of each band is a result of allowing $\gamma_{0}, \gamma_{\pi}, \alpha_{E 1}+\beta_{M 1}$, and $\alpha_{E 1}-\beta_{M 1}$, to vary by their experimental errors. It is clear
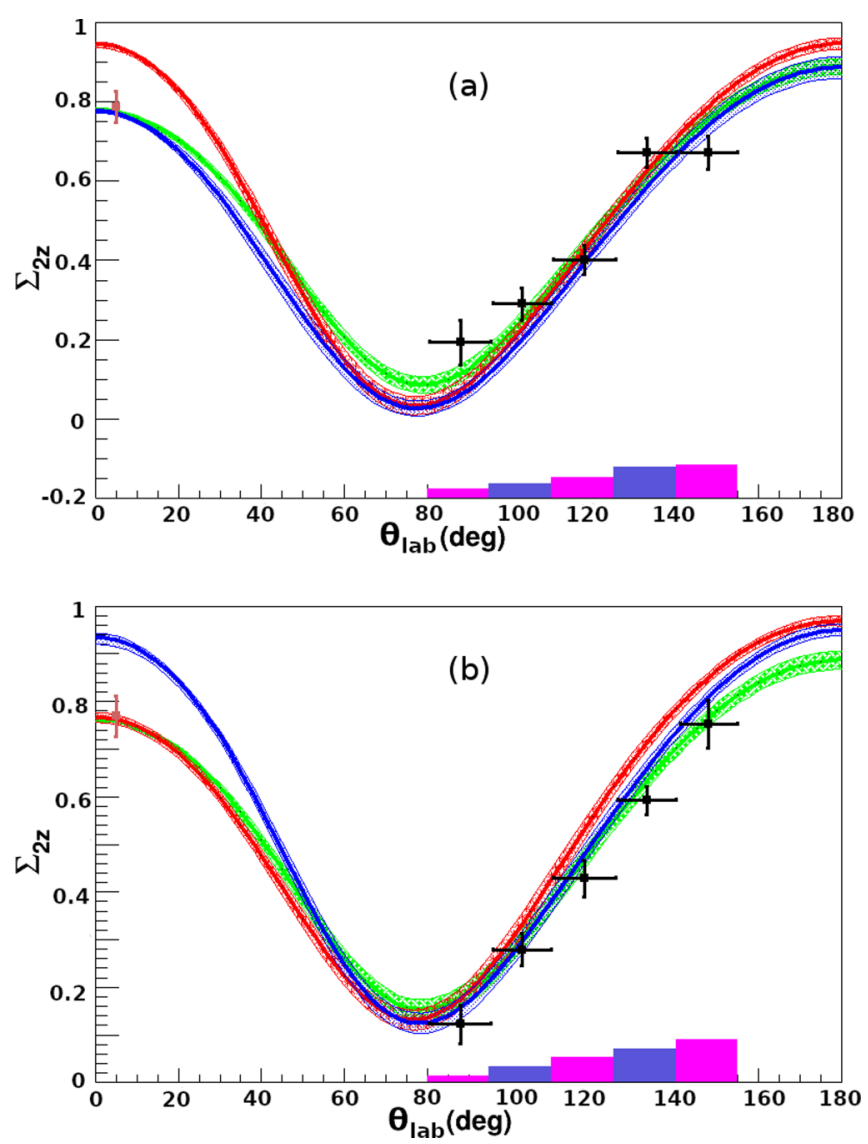

FIG. 5. Compton $\Sigma_{2 z}$ for $E_{\gamma}=265-285 \mathrm{MeV}$ (a) and $E_{\gamma}=$ 285-305 MeV (b). The red point is the value for $\Sigma_{2 z}$ at $0^{\circ}$, plotted at $5^{\circ}$ for readability, as determined by dispersive sum rules $[21,22]$. The green, red, and blue curves are from HDPV [2,23,24], B $\chi$ PT [25], and $\mathrm{HB} \chi$ PT $[26,27]$ calculations, respectively. For each, the central curve uses the weighted average values from Table II, and the width of each band represents the parameters varying within the errors quoted in the same table. The error bars shown are point-to-point statistical plus random systematic errors added in quadrature. The correlated systematic uncertainties are shown as a separate block above the horizontal axis for each Compton angle. 
from Fig. 4 that the $\Sigma_{2 z}$ data in this energy range indicate a sensitivity to $\gamma_{M}$ - of approximately \pm 2 in the standard units. Alternatively, $\gamma_{M-}$ can be fixed at $1.5 \times 10^{-4} \mathrm{fm}^{4}$ and $\gamma_{E-}$ set at $-5.5,-3.5$, or -1.5 in the same units. Unlike the previous case, $\Sigma_{2 z}$ in this energy range showed a weak sensitivity to $\gamma_{E-\text {. }}$

\section{GLOBAL FITS}

A global analysis of $\Sigma_{2 z}$ data from this measurement, along with the published $\Sigma_{2 x}$ and $\Sigma_{3}^{\text {LEGS }}$ results, and the prior values of $\gamma_{0}$ and $\gamma_{\pi}$, was performed to study the model dependence and extract the SPs. This was done by fitting the asymmetry data using the HDPV calculation $[2,23,24]$ and a B $\chi$ PT calculation [25]. The extracted SPs determined using each model are summarized in Table II. The fit with HDPV results in $\gamma_{E-}=$ $-2.74 \times 10^{-4} \mathrm{fm}^{4}$ and $\gamma_{M-}=1.4$, in the same units, similar to the values used for the theoretical bands in Fig. 4. The values from the two models are fairly consistent, and the best estimate of a central value is given by the weighted average in the last column of Table II. The errors for the weighted average values were conservatively taken as the larger of the two fits. These errors were chosen in favor of the weighted error, because the weighted errors assume the uncertainties in the theoretical calculations are uncorrelated, for which this paper makes no statement. The data are again shown in Fig. 5, now with theoretical calculations for HDPV [2,23,24], B $\chi$ PT [25], and $\mathrm{HB} \chi \mathrm{PT}[26,27]$, using the weighted average values for the SPs.

\section{SUMMARY}

In summary, model-dependent extractions of the SPs from a combined data fit of double- and single-polarized Compton scattering asymmetry results in the $\Delta(1232)$ resonance region are presented. These extracted SPs are also in good agreement with dispersion relation [2,23,24], baryon chiral perturbation theory [32], heavy baryon chiral perturbation theory [26,27], $K$-matrix theory [33], and chiral Lagrangian [34] predictions. Although the uncertainties in the SPs are significantly improved compared to previously reported results [6], forthcoming $\Sigma_{3}$ results from MAMI experiments [35] are expected to provide further improvements in the determination of these fundamental nuclear structure terms.

\section{ACKNOWLEDGMENTS}

The authors wish to acknowledge the excellent support of the accelerator group and operators of MAMI. We also wish to acknowledge and thank B. Pasquini and V. Pascalutsa for the use of their theory codes, as well as H. Grießhammer, J. McGovern, D. Phillips, and M. Vanderhaeghen for their theoretical support. This work was supported by the Natural Sciences and Engineering Research Council of Canada (NSERC, FRN: SAPPJ-2015-00023), the U.S. Department of Energy (Offices of Science and $\mathrm{Nu}-$ clear Physics, Awards No. DE-FG02-99-ER41110, No. DEFG02-88ER40415, No. DEFG02-01-ER41194, and No. DESC0014323) and National Science Foundation (Grants No. PHY-1039130, No. PHY-1714833, and No. OISE-1358175), Deutsche Forschungsgemeinschaft (SFB443, SFB/TR16, and SFB1044), the European Community-Research Infrastructure Activity under the FP6 "Structuring the European Research Area" program (Hadron Physics, Contract No. RII3-CT2004-506078), Schweizerischer Nationalfonds (Contract Nos. 200020-156983, 132799, 121781, 117601, and 113511), the U.K. Science and Technology Facilities Council (STFC 57071/1, 50727/1, ST/L00478X/1, and ST/L005824/1), and INFN (Italy).
[1] F. Hagelstein, R. Miskimen, and V. Pascalutsa, Prog. Part. Nucl. Phys. 88, 29 (2016).

[2] B. Pasquini, D. Drechsel, and M. Vanderhaeghen, Phys. Rev. C 76, 015203 (2007).

[3] J. Ahrens et al., Phys. Rev. Lett. 87, 022003 (2001).

[4] H. Dutz et al., Phys. Rev. Lett. 91, 192001 (2003).

[5] M. Camen et al., Phys. Rev. C 65, 032202(R) (2002).

[6] P. P. Martel et al., Phys. Rev. Lett. 114, 112501 (2015).

[7] G. Blanpied et al., Phys. Rev. C 64, 025203 (2001).

[8] K. H. Kaiser et al., Nucl. Instrum. Methods Phys. Res. A 593, 159 (2008).

[9] A. Jankowiak et al., Eur. Phys. J. A 28, 149 (2006).

[10] K. Aulenbacher et al., Nucl. Instrum. Methods Phys. Res. A 391, 498 (1997).

[11] V. Tioukine, K. Aulenbacher, and E. Riehn, Rev. Sci. Instrum. 82, 033303 (2011).

[12] D. Paudyal, Ph.D. thesis, University of Regina (2017), http://wwwa2.kph.uni-mainz.de/images/publications/phd/ Paudyal-PhD-thesis.pdf.

[13] J. C. McGeorge et al., Eur. Phys. J. A 37, 129 (2008).

[14] A. Thomas, Eur. Phys. J. Spec. Top. 198, 171 (2011).

[15] A. Starostin et al., Phys. Rev. C 64, 055205 (2001).
[16] C. M. Tarbert et al., Phys. Rev. Lett. 100, 132301 (2008).

[17] F. Afzal, Ph.D. thesis, University of Bonn (2019), http://hss.ulb. uni-bonn.de/2019/5551/5551.htm.

[18] D. Paudyal, G. Huber, P. P. Martel, and D. Hornidge, Technical Report, http://lichen.phys.uregina.ca/cbtaps/techreport_ pi02019.pdf.

[19] M. Unverzagt et al., Eur. Phys. J. A 39, 169 (2009).

[20] R. Novotny, IEEE Trans. Nucl. Sci 38, 379 (1991).

[21] O. Gryniuk, F. Hagelstein, and V. Pascalutsa, Phys. Rev. D 92 , 074031 (2015).

[22] O. Gryniuk, F. Hagelstein, and V. Pascalutsa, Phys. Rev. D 94, 034043 (2016).

[23] B. R. Holstein, D. Drechsel, B. Pasquini, and M. Vanderhaeghen, Phys. Rev. C 61, 034316 (2000).

[24] D. Drechsel, B. Pasquini, and M. Vanderhaeghen, Phys. Rep. C 378, 99 (2003).

[25] V. Lensky and V. Pascalutsa, Eur. Phys. J. C 65, 207 (2010)

[26] H. W. Grießhammer, J. A. McGovern, and D. R. Phillips, Eur. Phys. J. A 52, 139 (2016).

[27] H. W. Grießhammer, J. A. McGovern, and D. R. Phillips, Eur. Phys. J. A 54, 37 (2018). 
[28] V. Olmos de León et al., Eur. Phys. J. A 10, 207 (2001).

[29] M. Tanabashi et al., Phys. Rev. D 98, 030001 (2018).

[30] H. W. Grießhammer, J. A. McGovern, D. R. Phillips, and G. Feldman, Prog. Part. Nucl. Phys. 67, 841 (2012).

[31] B. Pasquini, P. Pedroni, and S. Sconfietti, J. Phys. G: Nucl. Part. Phys. 46, 104001 (2019).
[32] V. Lensky, J. A. McGovern, and V. Pascalutsa, Eur. Phys. J. C 75, 604 (2015).

[33] S. Kondratyuk and O. Scholten, Phys. Rev. C 64, 024005 (2001).

[34] A. M. Gasparyan, M. F. M. Lutz, and B. Pasquini, Nucl. Phys. A 866, 79 (2011).

[35] C. Collicott et al. (in preparation, 2020). 